\title{
A comparison of light spot hydrophone and fiber optic probe hydrophone for lithotripter field characterization
}

\author{
N. Smith, ${ }^{1, \text { a) }}$ G. N. Sankin, ${ }^{1}$ W. N. Simmons,${ }^{1}$ R. Nanke ${ }^{2}$ J. Fehre, ${ }^{2}$ and P. Zhong ${ }^{1}$ \\ ${ }^{1}$ Department of Mechanical Engineering and Materials Science, Duke University, \\ Durham, North Carolina 27708, USA \\ ${ }^{2}$ Research and Development Urology, Siemens AG, Healthcare Sector, 91052 Erlangen, Germany
}

(Received 31 August 2011; accepted 3 January 2012; published online 30 January 2012)

\begin{abstract}
The performance of a newly developed light spot hydrophone (LSHD) in lithotripter field characterization was compared to that of the fiber optic probe hydrophone (FOPH). Pressure waveforms produced by a stable electromagnetic shock wave source were measured by the LSHD and FOPH under identical experimental conditions. In the low energy regime, focus and field acoustic parameters matched well between the two hydrophones. At clinically relevant high energy settings for shock wave lithotripsy, the measured leading compressive pressure waveforms matched closely with each other. However, the LSHD recorded slightly larger IP_I $(p<0.05)$ and secondary peak compressive pressures $(p<0.01)$ than the FOPH, leading to about $20 \%$ increase in total acoustic pulse energy calculated in a $6 \mathrm{~mm}$ radius around the focus $(p=0.06)$. Tensile pulse durations deviated $\sim 5 \%$ $(p<0.01)$ due to tensile wave shortening from cavitation activity using the LSHD. Intermittent compression spikes and laser light reflection artifacts have been correlated to bubble activity based on simultaneous high-speed imaging analysis. Altogether, both hydrophones are adequate for lithotripter field characterization as specified by the international standard IEC 61846. (O) 2012 American Institute of Physics. [doi:10.1063/1.3678638]
\end{abstract}

\section{INTRODUCTION}

Accurate calibration of lithotripter fields requires a hydrophone with large dynamic range (peak pressures varying from $1 \mathrm{MPa}$ to $150 \mathrm{MPa}$ ), broad frequency bandwidth (>30 MHz), and high spatial resolution $(<0.2 \mathrm{~mm}){ }^{1,2}$ The strong compressive shock front and rarefaction tail characteristics of lithotripter pulses demand a durable, long-lasting hydrophone with high water-surface adhesion to reduce cavitation damage and measurement artifacts. ${ }^{3,4}$ In addition, a desirable hydrophone should be compact and convenient for a variety of measurements in shock wave lithotripsy (SWL) and other therapeutic applications of high energy shock waves.

For many years, the polyvinylidene fluoride (PVDF) hydrophones represented the standard for measuring ultrasonic fields. ${ }^{5}$ The flat frequency response to an incident acoustic wave and high durability of the PVDF hydrophone provided significant advantages over hydrophones made of piezoceramic crystal elements. ${ }^{6-8}$ In particular, PVDF needle hydrophones have been used in lithotripter field characterization because of their near omnidirectional sensitivity and reasonable spatial resolution. ${ }^{8}$ However, "fringe" field interference limits further reductions in sensor element size of PVDF needle hydrophones. ${ }^{5}$ PVDF susceptibility to cavitation damage, inadequacy in resolving the strong tensile components in lithotripter-generated shock waves, and frequent recalibration due to thermal depolarization of the polymer ultimately led to the advent of new hydrophone technologies. ${ }^{3,5,9}$

The fiber optic probe hydrophone (FOPH), introduced in $1988,{ }^{10}$ improves upon PVDF hydrophones by using

\footnotetext{
a) Author to whom correspondence should be addressed. Electronic mail: nathan.b.smith@duke.edu. Tel.: 919-660-5176.
}

highly water-adhesive silica glass fiber $(\varnothing=143 \mu \mathrm{m})$ for laser light transmission to and from its sensor tip. Fresnel reflection of light relates to pressure fluctuations in water at the fiber tip through the Gladstone-Dale relationship of refractive index and density, and correlates to pressure via the Tait equation of state in water. ${ }^{3}$ Many optical hydrophone variants have been developed, ${ }^{11-17}$ though the FOPH continues to be the most prevalently used for lithotripter field characterization. ${ }^{18-21}$ Recent applications include shock wave-bubble interaction, ${ }^{22-24}$ cavitation impact assessment, ${ }^{25,26}$ and pressure characterization near to the surface of phantom kidney stones. ${ }^{27}$

Despite its widespread use, the FOPH has two salient drawbacks: tip damage will lead to the loss of measurement position and diffraction effects require signal deconvolution for accurate calibration. These limitations have motivated the creation of an alternative optical hydrophone, namely, the light spot hydrophone (LSHD), which operates under the same physical principles as the FOPH. Introduced in $2004,{ }^{28}$ the LSHD utilizes two fiber-optic lens assemblies confocally aligned to a small spot $(\varnothing=100 \mu \mathrm{m})$ on a large glass block $(\mathrm{L} \times \mathrm{W} \times \mathrm{H}=90 \mathrm{~mm} \times 60 \mathrm{~mm} \times 30 \mathrm{~mm}$ ), which acts as the interface for pressure measurements in a fluid. In many ways, the LSHD is an extension of the optical stress transducer developed by Paltauf et al. ${ }^{29,30}$ Laser light transmitted to the glass-fluid interface is reflected and recorded by a photodetector, and the received signal is then converted to pressure off-line based on pressure-density-refraction index relationships. ${ }^{3,28}$

In this study, we compare the performance of the FOPH and LSHD in lithotripter field characterization for both low and clinically applicable source energy settings, to be distinguished from previous fiber optic-style hydrophone 

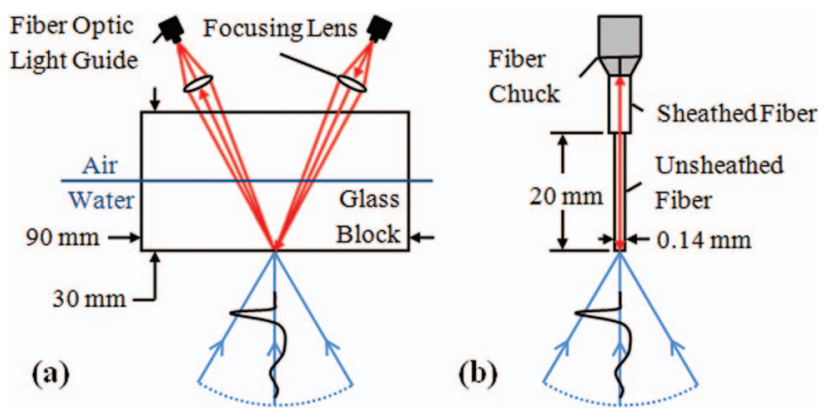

FIG. 1. (Color online) A schematic diagram of the (a) LSHD and (b) FOPH and their principles of operation.

and LSHD comparisons at a single spatial position and source energy. ${ }^{14,31}$ Interpretation is offered for differences in lithotripter shock wave (LSW) parameters measured with each hydrophone and the roles of cavitation in measurement interference. Advantages and disadvantages related to each hydrophone's usage are discussed with considerations for general applicability in SWL.

\section{MATERIALS AND METHODS}

\section{A. Shock wave source and hydrophones}

Using a highly stable experimental electromagnetic (EM) shock wave generator with a focal length of $140 \mathrm{~mm}$ and aperture diameter of $125 \mathrm{~mm}$, two identical sets of shock wave measurements were conducted in succession with a light spot hydrophone (LSHD-2, University of Erlangen-Nuremberg, Germany) and a fiber optic probe hydrophone (FOPH 500, RP Acoustics, Leutenbach, Germany). Figure 1 illustrates schematically the principles of operation at the glass-water interfaces for the LSHD and FOPH.

Table I summarizes the specifications of the hydrophones based on data either provided by the respective manufacturers or extracted from publications. ${ }^{3,28}$

The LSHD presented in this study is an upgraded model with reduced low frequency (LF) cutoff from models previously reported. ${ }^{14,28,31}$ The spatial resolutions of the LSHD and FOPH are identical. LSHD sensitivity $\left(H_{\mathrm{LSHD}}\right)$ is boosted electronically through the use of dc-compensating transimpedance amplification $\left(Z_{T, \text { ac }} / Z_{T, \text { dc }}\right)$ of the return optical signal, as shown in Eq. (1):

$$
H_{\mathrm{LSHD}}(p)=\left.\left.U_{\mathrm{dc}} \frac{d R_{G W}}{d p}\right|_{C_{R} \mathrm{p}} \frac{1}{R_{G W}}\right|_{\mathrm{p}=0} C_{R} \frac{Z_{T, \mathrm{ac}}}{Z_{T, \mathrm{dc}}} .
$$

TABLE I. Specifications of the light spot hydrophone (LSHD) and fiber optic probe hydrophone (FOPH).

\begin{tabular}{lcc}
\hline \hline Properties & LSHD & FOPH \\
\hline Bandwidth & $1.5-40 \mathrm{MHz}$ & $0-50 \mathrm{MHz}^{\mathrm{a}}$ \\
Sensitivity & $10 \mathrm{mV} / \mathrm{Mpa}$ & $2 \mathrm{mV} / \mathrm{MPa}$ \\
Spatial resolution & $100 \mu \mathrm{m}$ & $100 \mu \mathrm{m}$ \\
Laser light wavelength & $780 \mathrm{~nm}$ & $810 \mathrm{~nm}$ \\
Laser power & $35 \mathrm{~mW}$ & $500 \mathrm{~mW}$ \\
\hline \hline
\end{tabular}

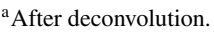

The optical reflectivity $\left(R_{G W}\right)$ and dc baseline voltage $\left(U_{\mathrm{dc}}\right)$ depend slightly on laser light polarization state due to the angle of incidence $\left(=6.86^{\circ}\right.$ relative to glass surface normal); it has been determined that optical alignment variation and polarization state dependence account for $<0.2 \%$ error when measurements are normalized to $U_{\mathrm{dc}}$. Due to the absence of edge diffraction effects over the duration of a typical LSW measurement $(\sim 20 \mu \mathrm{s})$, the "doubling" of acoustic pressure $(p)$ from wave reflection at the glass-water interface can be incorporated directly in the LSHD sensitivity formula. $C_{R}$ $(=1.79)$ is the acoustic pressure reflection factor of the glasswater interface. In comparison, the FOPH uses a deconvolution program to correct for contributions from fiber edge diffraction: ${ }^{4}$

$$
H_{\mathrm{FOPH}}(\mathrm{p})=\left.U_{\mathrm{dc}} \frac{d R_{G W}}{d \mathrm{p}} \frac{1}{\left(R_{G W}+\alpha\right)}\right|_{\mathrm{p}=0} .
$$

The FOPH sensitivity $\left(H_{\mathrm{FOPH}}\right)$ in Eq. (2) includes a light scattering factor $(\alpha=0.04)$ to adjust for fiber splitting and optical fiber losses. Moreover, imperfect preparations, dust contamination, and general wear and tear contribute to reduce $U_{\mathrm{dc}}$ and sensitivities in both hydrophones. In all the tests described hereafter, the $\operatorname{LSHD}\left(H_{\mathrm{LSHD}}(0)=-6.4 \mathrm{mV} / \mathrm{MPa}\right)$ and FOPH $\left(H_{\mathrm{FOPH}}(0)=-1.3 \mathrm{mV} / \mathrm{MPa}\right)$ were operated above acceptable baseline voltages specified by the respective manufacturers.

\section{B. Experimental setup and pressure measurement protocol}

As shown in Fig. 2, the EM shock wave generator was mounted at the bottom of a Lucite tank $(\mathrm{L} \times \mathrm{W} \times \mathrm{H}=40 \mathrm{~cm}$ $\times 40 \mathrm{~cm} \times 30 \mathrm{~cm}$ ) filled with $0.2 \mu \mathrm{m}$-filtered and $19 \pm 0.5^{\circ} \mathrm{C}$ degassed water. The oxygen concentration in the water tank was $\leq 1.5 \mathrm{mg} / \mathrm{l}$ to start and increased by $<1.25 \mathrm{mg} / \mathrm{l}$ within the duration of each experiment. The hydrophones were attached to a 3D positioning system (VXM-2 step motors with BiSlide-M02 lead screws, Velmex, Bloomfield, NY) with a minimum step size of $5 \mu \mathrm{m}$ and a maximum scan range of

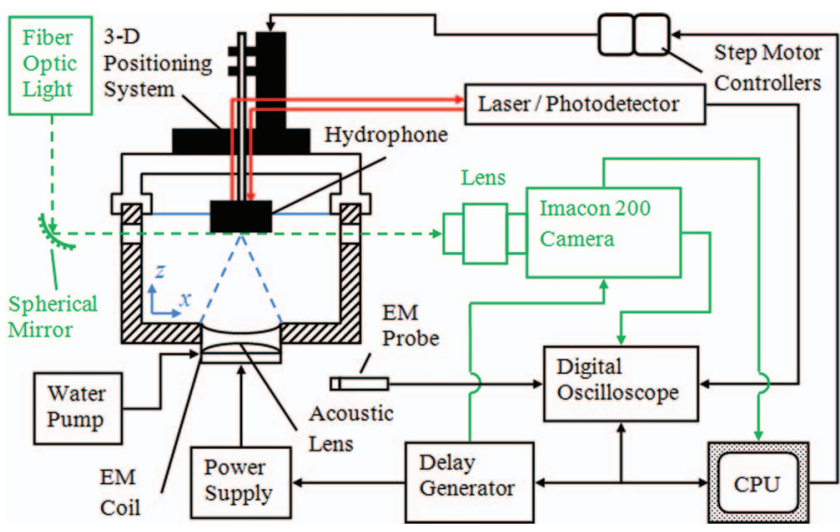

FIG. 2. (Color online) A schematic diagram of the experimental setups for pressure mapping and high speed imaging, drawn for illustrative purposes with the LSHD set at the focus of the EM shock source. 
$250 \mathrm{~mm}$. Hydrophone alignment and axial scan procedure were controlled remotely using a LABVIEW (National Instruments, Austin, TX) program. At each field position, a single shock wave was measured by the hydrophone and recorded on a digital oscilloscope (LeCroy 6050A, Chestnut Ridge, NY) operated at a sampling rate of $100 \mathrm{MHz}$. Multiple samples at each measurement point were obtained through iterations of the scans. A slow pulse repetition frequency (PRF) of $0.05 \mathrm{~Hz}$ was used to ensure minimal cavitation interference on the pressure waveform..$^{32,33}$

To remove potential cavitation nuclei around the fiber, pre-conditioning of the FOPH was performed following a protocol described previously. ${ }^{25}$ Beginning at a low output energy, shock waves were fired at a low $\operatorname{PRF}(=0.33 \mathrm{~Hz})$ until waveforms were consistent and reproducible. The output energy of the shock source was then increased incrementally until reaching a high energy setting for precise hydrophone alignment. Without pre-conditioning, the fiber tip would break easily, resulting in loss of spatial reference prior to completion of the field characterization. Similar preparations were not required with the LSHD; however, frequent brushing of the glass surface during alignment helped maintain clean measurements and protected the glass block from cavitation damage.

\section{Data processing and analysis}

Pressure conversion of the FOPH was performed using a Windows program CALDEC.exe (RP Acoustics, Leutenbach, Germany) that consists of four main steps: (1) dc-normalized photovoltage to pressure conversion based on the calibration equation; (2) Fourier transformation of the pressure-time data into the frequency domain; (3) division of the measured signal by the provided FOPH transfer function; and (4) inverse Fourier transformation of the corrected signal to the time domain. In comparison, pressure conversion in the LSHD was carried out using a one-step algorithm written in MATLAB (Mathworks, Natick, MA), based on a third-order calibration polynomial. The calibration equation is an approximation (accurate to with $1 \%$ for the pressure range in this study) of the formula derived from aforementioned physical principles. ${ }^{3,28}$ Additionally, the LSHD employs a separate path for dc baseline monitoring that allows the user to calibrate unambiguously from the baseline signal captured simultaneously with the measured acoustic signal. FOPH baseline voltages were monitored on a single path pre- and post-measurement. Both hydrophone calibrations account for silica glass compressibility. The LSHD calibration does not include water temperature corrections.

Post-processing of the calibrated data for both hydrophones was conducted in MATLAB. All experimental results involving averaged waveforms were carried out by aligning the 10\%-peak amplitude on the ascending shock front. Typical shock wave parameters, such as peak pressures $\left(\mathrm{P}_{+},\left|\mathrm{P}_{-}\right|\right)$, pulse durations $\left(t_{+}, t_{-}\right)$, full width half maximum (FWHM) beam sizes, and pulse-intensity integrals were calculated based on IEC 61846 lithotripter characterization protocol. The total acoustic energy is expressed as

$$
E=\frac{1}{Z_{W}} \iint_{A, t} p^{2} d A d t,
$$

where $p$ is the acoustic pressure measured by each hydrophone and $Z_{W}$ is the acoustic impedance in water. Energy integration bounds in time $(t)$ were designated by first and final crossing points of the 10\%-local peak pressure level, and in space by a circular cross-sectional area $(A)$ of radius $6 \mathrm{~mm}$, an area encompassing most stones treated with extracorporeal shock wave lithotripsy. Total acoustic energy was corrected over the integration bounds by subtraction of averaged hydrophone noise contributions.

\section{High-speed imaging of bubble activities near the hydrophone sensor}

To assess the fidelity of hydrophone signal, cavitation activity in the focal volume was recorded using an intensified charge-coupled device high-speed video camera (Imacon 200, DRS Hadland) equipped with a Micro-NIKKOR $55 \mathrm{~mm}$ f/2.8 lens (Nikon). Light from a ML-1000 fiber-optic coupled Xenon flashlamp (Dyna-Lite) was collimated by a spherical mirror $(f=4 \mathrm{ft})$ and projected through the lithotripter focal region to the camera. Fourteen shadowgraphic images were captured at a framing rate of 1 million frames/s with $250 \mathrm{~ns}$ in exposure time prior to and shortly after the arrival of the shock wave. Trigger output from the camera was recorded to provide the precise timing sequence of the high-speed images for comparison with the hydrophone signal measured concomitantly.

\section{RESULTS AND DISCUSSION}

\section{A. Pressure waveforms at geometric focus of the shock source}

Figures 3(a) and 3(b) show averaged pressure waveforms $(\mathrm{n}=12)$ at two different energy settings. Characteristic parameters of the acoustic field calculated from these waveforms are summarized in Table II.

At a low energy setting (which corresponds to a source voltage of $9.5 \mathrm{kV}$ ), the pressure waveforms measured by FOPH and LSHD match closely with each other. At a high energy setting (i.e., $15.8 \mathrm{kV}$ ), there is no statistical difference in measured peak positive pressures $\left(\mathrm{P}_{+}\right)$; however, peak negative pressures $\left(\mathrm{P}_{-}\right)$deviate by about $10 \%(p<0.05)$. Focal peak pressure measurements $(n=4)$ from Fig. 3(c) confirm these results over a range of lithotripter energy settings, including measurements at the dynamic limit of the shock wave generator (i.e., $19 \mathrm{kV}$ ). The LSHD photovoltage signal-tonoise ratio at a high energy setting is $22 \mathrm{~dB}$ (cf. $24 \mathrm{~dB}$ for the FOPH); high frequency noise ( $>30 \mathrm{MHz}$ ) has been filtered out in tabulated values of $\mathrm{P}_{-}$.

Differences in characterization parameters may result from electronic filtration, particularly in the low frequency spectrum. The respective LSHD and FOPH bandwidths are presented in Table I. In Fig. 4, representative LSHD 


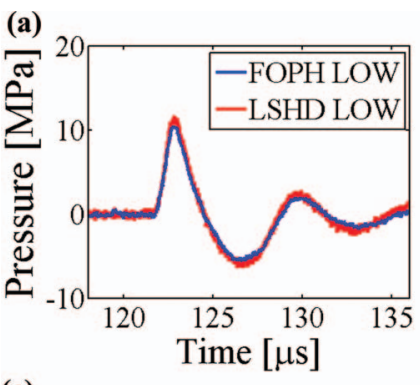

(b)

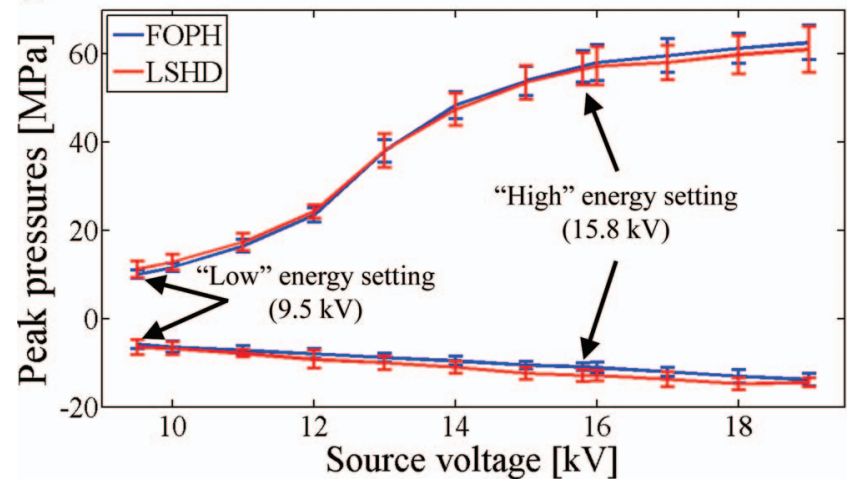

FIG. 3. (Color online) Representative averaged $(n=12)$ pressure waveforms of the LSHD (light) and FOPH (dark) at (a) low energy and (b) high energy settings in the shock source geometric focus. (c) Averaged peak compressive and tensile pressures $(n=4)$ at the focus for a range of relevant shock source voltages.

amplifier filtration (i.e., low frequency cutoffs $=1.5 \mathrm{kHz}$ and $15 \mathrm{kHz}$ ) applied to FOPH photovoltage data shows how bandwidth limitation can both alter pulse durations and increase $\mid \mathrm{P}_{\text {_l}}$. At $1.5 \mathrm{kHz}$ low frequency cutoff, waveform distortion is nearly negligible, though at $15 \mathrm{kHz}$, the effect is pronounced. In this way, measurement errors may be a side effect of higher sensitivity with the LSHD. Previous studies comparing optical probe hydrophones with the LSHD do not discuss this effect, though it may be seen when measurements are normalized to ambient pressure. ${ }^{14,31}$

As shown in Fig. 3(b) and Table II, LSHD measurements of tensile pulse duration $\left(t_{-}\right)$and secondary pressure deviate from FOPH measurements at a high energy setting $(p<0.01)$. These effects are reminiscent of acoustic radiation amassment from inertial cavitation near to a FOPH fiber tip observed in previous studies implementing seeded bubbles, tap water, and/or strong IP_I (>40 MPa). ${ }^{24,26,34-36}$ High-speed imaging in Fig. 5(a) suggests that cavitating bubbles formed in a thin layer beneath the light spot may likewise alter the measured

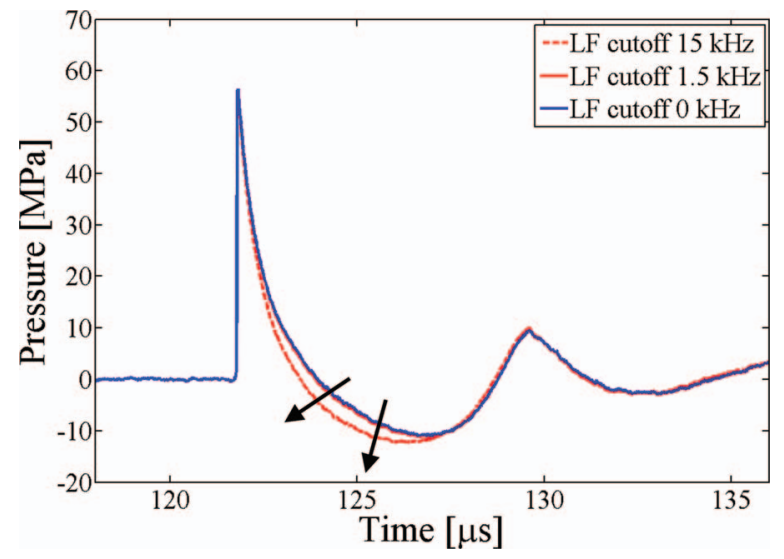

FIG. 4. (Color online) FOPH averaged $(n=12)$ pressure waveform postprocessed through a digital 2nd-order Butterworth high-pass filter with LF cutoff of $15 \mathrm{kHz}$ (dashed/light), $1.5 \mathrm{kHz}$ (solid/light), and $0 \mathrm{kHz}$ (solid/dark). The LF cutoff of $15 \mathrm{kHz}$ of LSHD data previously reported is represented. ${ }^{14,31}$ Filter applied to individual photovoltage measurements prior to pressure calibration and averaging.

pressure waveform. An incident LSW and associated bubble activity produced near the tip of the FOPH is shown in Fig. 5(b), which resemble that of the free field.

\section{B. Pressure distribution}

The representative focal plane map of Fig. 6(a) and acoustic propagation ( $z$ ) axis measurements of Fig. 6(b) correspond well between the LSHD and FOPH within measurement errors. No statistical difference is seen in $\mathrm{P}_{+}$values at any acoustic field position or source energy setting $(n=4)$. Statistically higher $\left|\mathrm{P}_{-}\right|(p \leq 0.05)$ measured by the LSHD occurs primarily at a high energy setting and along the focal plane axes (20 out of 41 total field positions).

No statistical differences were observed in the FWHM focal width and beam extent of the lithotripter field both at low and high energy output settings shown in Table III. The tabulated total energy values for the FOPH $\left(\mathrm{E}_{\mathrm{LOW}}=10 \mathrm{~mJ}\right.$; $\left.\mathrm{E}_{\mathrm{HIGH}}=75 \mathrm{~mJ}\right)$ and LSHD $\left(\mathrm{E}_{\mathrm{LOW}}=12 \mathrm{~mJ} ; \mathrm{E}_{\mathrm{HIGH}}=90 \mathrm{~mJ}\right)$ differ by $\sim 20 \%$ at both energy settings ( $p \geq 0.06$ ). Tensile energy differences at the high energy setting $(p<0.05)$ suggest that predominantly, LSHD energy contributions from higher IP_I offset a shorter tensile pulse duration. The impact of bubble activity is unambiguous, however, noting the statistically higher LSHD second compressive energy at the high energy setting $(p<0.01)$.

TABLE II. Lithotripsy parameters derived from measurements by the LSHD and FOPH, including peak pressures and pulse durations. Error is standard deviation in measurement plus uncertainties (in pressure or propagated uncertainties) provided by the respective manufacturers.

\begin{tabular}{lccccc}
\hline \hline Lithotripsy parameters & & $\mathrm{P}_{+}(\mathrm{MPa})$ & $\mathrm{P}_{-}(\mathrm{MPa})$ & $t_{+}(\mu \mathrm{s})$ & $t_{-}(\mu \mathrm{s})$ \\
\hline Low energy & LSHD & $11.1 \pm 1.6$ & $-6.0 \pm 1.3$ & $2.3 \pm 0.2$ & $4.1 \pm 0.3$ \\
& FOPH & $10.5 \pm 1.1$ & $-5.6 \pm 0.7$ & $2.4 \pm 0.3$ & $4.1 \pm 0.3$ \\
High energy & LSHD & $55.7 \pm 3.8$ & $-12.5 \pm 1.7^{\mathrm{a}}$ & $1.5 \pm 0.2$ & $4.3 \pm 0.3^{\mathrm{b}}$ \\
& FOPH & $56.4 \pm 3.8$ & $-11.1 \pm 1.2$ & $1.6 \pm 0.3$ & $4.6 \pm 0.2$ \\
\hline \hline
\end{tabular}

${ }^{\mathrm{a}} p<0.05$.

${ }^{\mathrm{b}} p<0.01$. 


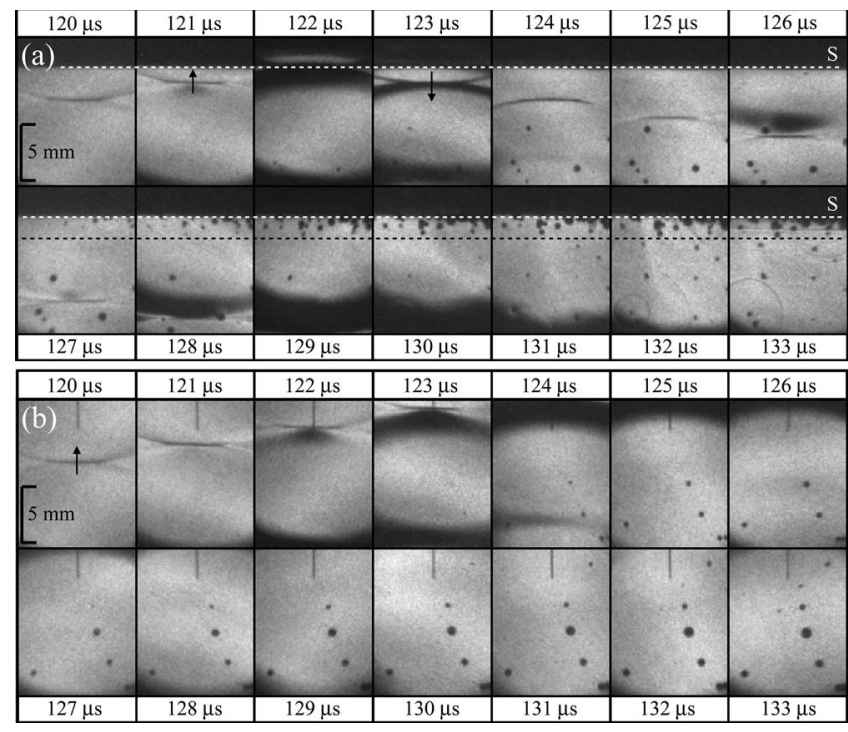

FIG. 5. Representative bubble activity for the (a) LSHD and (b) FOPH during a single high energy LSW measurement. (Arrows over LSW indicate its propagation direction. "S" marks the surface of the LSHD glass block. A black dashed line shows the layer of rapid bubble growth adjacent to the glass block).

\section{Cavitation-related artifacts}

\section{Acoustic pressure spikes}

LSW reflection off the large LSHD glass surface creates bubbly phenomena not typically present in the approximated free-field of the FOPH. Sharp compression spikes of several hundred nanosecond duration are one such disturbance that can be superimposed onto the LSHD-measured waveform. Typically, these appear in and beyond the second compression phase of measurements at a high energy setting. In instances where suspended bubbles pre-exist near to the light spot prior to measurement, compression spikes may appear at any point after LSW arrival. It is believed these short-lived shocks are caused by the forced collapse of microbubbles. ${ }^{37}$ Similar FOPH measurements have been seen in laser-induced single bubble and near-boundary bubble collapse studies. ${ }^{22,27}$

Inertial cavitation occurs in banded layers below the LSHD glass block, similar to bubble dynamics induced by intersecting lithotripter shock waves. ${ }^{38,39}$ Three distinct layers develop in the immediate wake of a LSW. First, in a narrow ( $\sim 2 \mathrm{~mm}$ wide) layer nearest to the LSHD glasswater surface, microbubbles grow rapidly from constructive overlap of the incident and reflected tensile pressure. This phenomenon correlates to LSW parameters such as $t_{-}$and
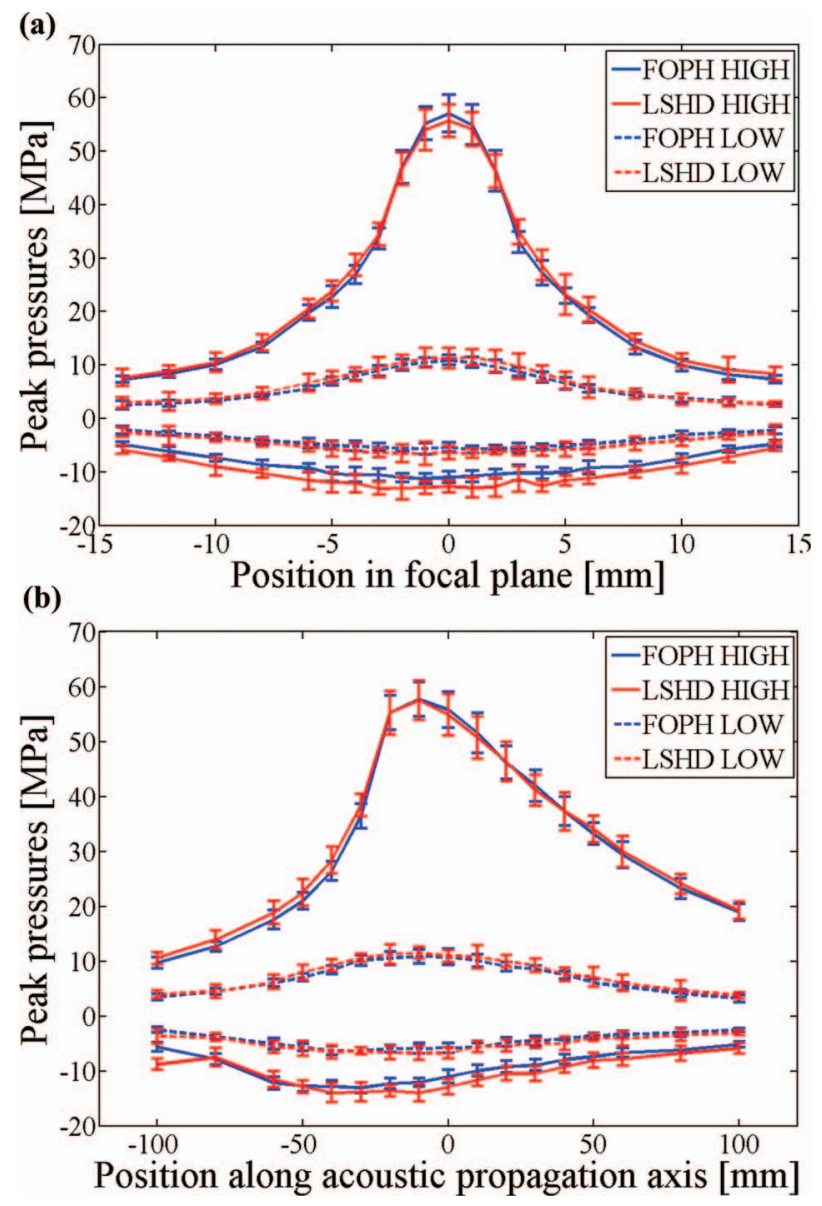

FIG. 6. (Color online) Pressure maps of averaged peak compressive and tensile pressures $(n=4)$ along (a) one focal plane axis and (b) the acoustic propagation ( $z$ ) axis using the LSHD (light) and FOPH (dark) at both high and low shock source energies (solid and dashed, respectively). Only one focal plane axis is shown because of lithotripter source symmetry.

$\mathrm{P}_{-}$, and complies with theoretical work on cavitation enhancement near an infinite rigid boundary. ${ }^{39} \mathrm{~A}$ second layer below the first $(\sim 3 \mathrm{~mm}$ wide) experiences inappreciable cavitation due to destructive wave interference. ${ }^{40}$ Beginning at an estimated distance of $5 \mathrm{~mm}$ from the glass block, bubbles visibly grow prior to the arrival of reflected $\mathrm{P}_{+}$. Forced bubble collapse may occur when $\mathrm{P}_{+}$passes through this layer, in some instances superposing secondary compression of the approaching LSW. Spherical collapse shocks can broaden and strike the light spot should clear transmission lines to the light spot exist. Figures 7(a) and 7(b) illustrate this hydrophone measurement artifact and its corresponding

TABLE III. Lithotripsy parameters derived from measurements by the LSHD and FOPH, which include full-width half-maximum (-6 dB) focal width and extent, and acoustic energies: first compressive $\left(\mathrm{E}_{+1}\right)$, first tensile $\left(\mathrm{E}_{-1}\right)$, second compressive $\left(\mathrm{E}_{+2}\right)$, and total $\left(\mathrm{E}_{\mathrm{TOT}}\right)$.

\begin{tabular}{|c|c|c|c|c|c|c|c|}
\hline Lithotripsy parameters & & Focal width (mm) & Focal extent $(\mathrm{cm})$ & $\mathrm{E}_{+1}(\mathrm{~mJ})$ & $\mathrm{E}_{-1}(\mathrm{~mJ})$ & $\mathrm{E}_{+2}(\mathrm{~mJ})$ & $\mathrm{E}_{\mathrm{TOT}}(\mathrm{mJ})$ \\
\hline \multirow[t]{2}{*}{ Low energy } & LSHD & $12.1 \pm 3.1$ & $12.9 \pm 1.8$ & $6.7 \pm 0.9$ & $5.1 \pm 0.8$ & $0.1 \pm 0.0$ & $11.9 \pm 1.4$ \\
\hline & FOPH & $12.5 \pm 1.7$ & $13.0 \pm 1.9$ & $5.7 \pm 0.6$ & $4.1 \pm 0.5$ & $0.2 \pm 0.0$ & $10.0 \pm 1.0$ \\
\hline \multirow[t]{2}{*}{ High energy } & LSHD & $8.4 \pm 1.1$ & $10.7 \pm 1.4$ & $56.4 \pm 6.1$ & $25.2 \pm 4.2^{\mathrm{a}}$ & $8.4 \pm 1.7^{\mathrm{b}}$ & $90.4 \pm 10.8$ \\
\hline & FOPH & $7.5 \pm 1.1$ & $10.0 \pm 1.4$ & $51.7 \pm 5.4$ & $18.3 \pm 2.2$ & $4.3 \pm 0.9$ & $74.7 \pm 8.5$ \\
\hline
\end{tabular}

${ }^{\mathrm{a}} p<0.05$.

${ }^{\mathrm{b}} p<0.01$. 

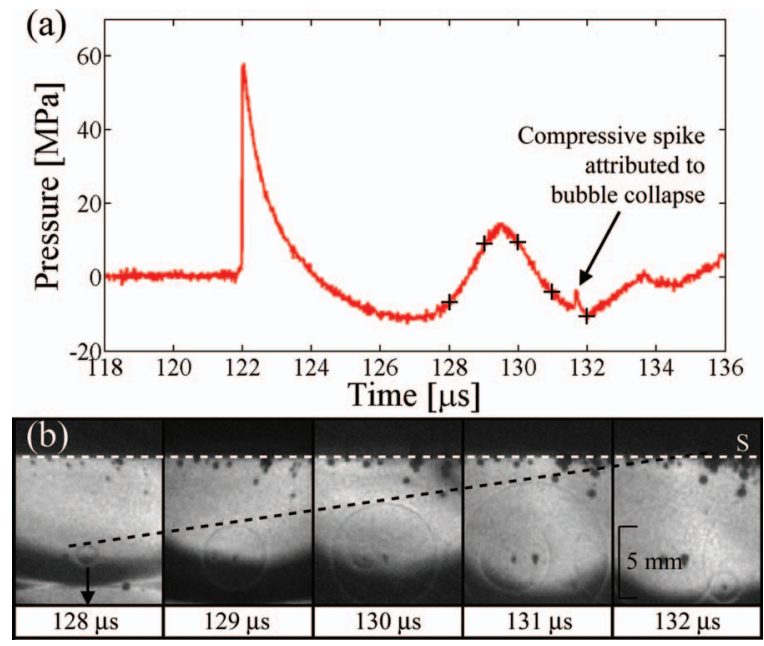

FIG. 7. (Color online) (a) Representative LSHD waveform with bubble collapse spike and 5 Imacon 200 camera exposures $(+)$ indicated on waveform. (b) Corresponding frames of LSHD bubble activity tracing a single bubble's collapse (black dashed line) to its arrival at the glass block surface "S." Arrow over LSW indicates its propagation direction.

bubble activity, respectively. Depending on bubble proximity to the sensitive area, strength of collapse, and attenuation from other bubbles, these spherical shocks have been witnessed to crest on the order of $\mathrm{P}_{+}$.

\section{Bubble at laser spot}

Photodetector saturation from inertial bubble growth at the fiber tip is a well documented artifact in FOPH shock wave measurements. ${ }^{18,27,31,41,42}$ Similar interference by inertial bubbles was observed near the LSHD laser spot. Hydrophone measurements of Fig. 8(a) in tandem with high-speed images of bubble activity in Figs. 8(b) and 8(c) demonstrate how bubble expansion at the respective sensitive areas can cause an order of magnitude change in reflected laser light and a positive spike in recorded voltage signal (which corresponds to a negative spike in pressure). Using the LSHD, this effect can be seen through simultaneous dc volt-
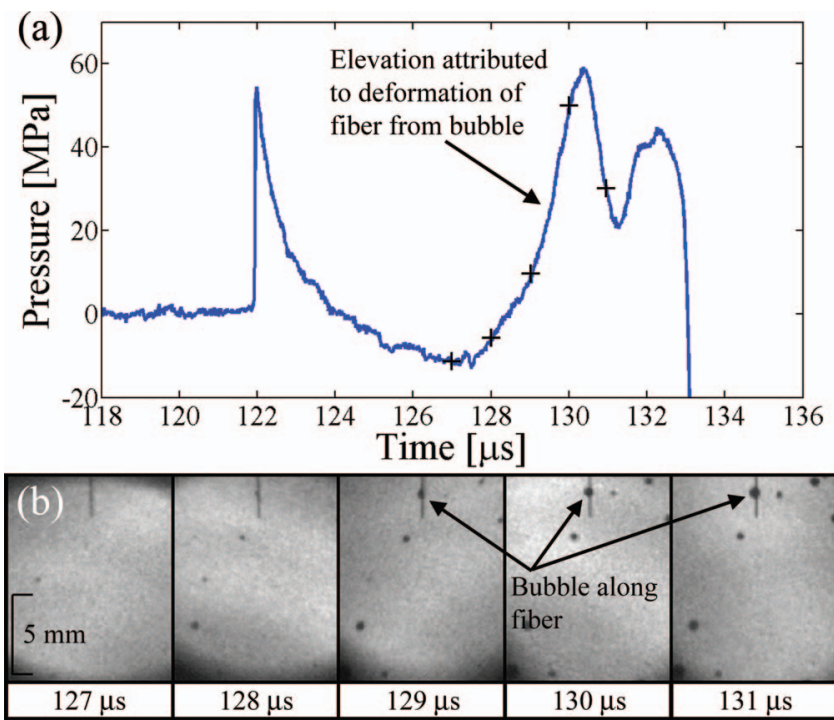

FIG. 9. (Color online) (a) Representative FOPH waveform corrupted by bubble distortion of fiber and 5 Imacon 200 camera exposures $(+)$ indicated on waveform. (b) Corresponding frames of FOPH bubble activity.

age and signal output changes. As shown in the final frame of Fig. 8(c), the LSHD measurement spot coincides in the imaging plane with a bubble on the glass block surface.

\section{Fiber deformation from bubble}

In previous studies, a fiber deformation mechanism has been proposed to account for decreased laser light in FOPH measurement. ${ }^{18}$ This artifact is unique to the FOPH. It typically follows the first tensile wave and supervenes on the second compressive wave, prohibiting the signal from returning to zero after the passage of the LSW. Figures 9(a) and 9(b) demonstrate this waveform distortion, which is highly sensitive to fiber preparation quality and appears to be initiated by Harvey nuclei ${ }^{43}$ Fiber pre-conditioning helps reduce measurement interference from this artifact, ${ }^{25}$ and may also produce local degassing effects. ${ }^{44,45}$
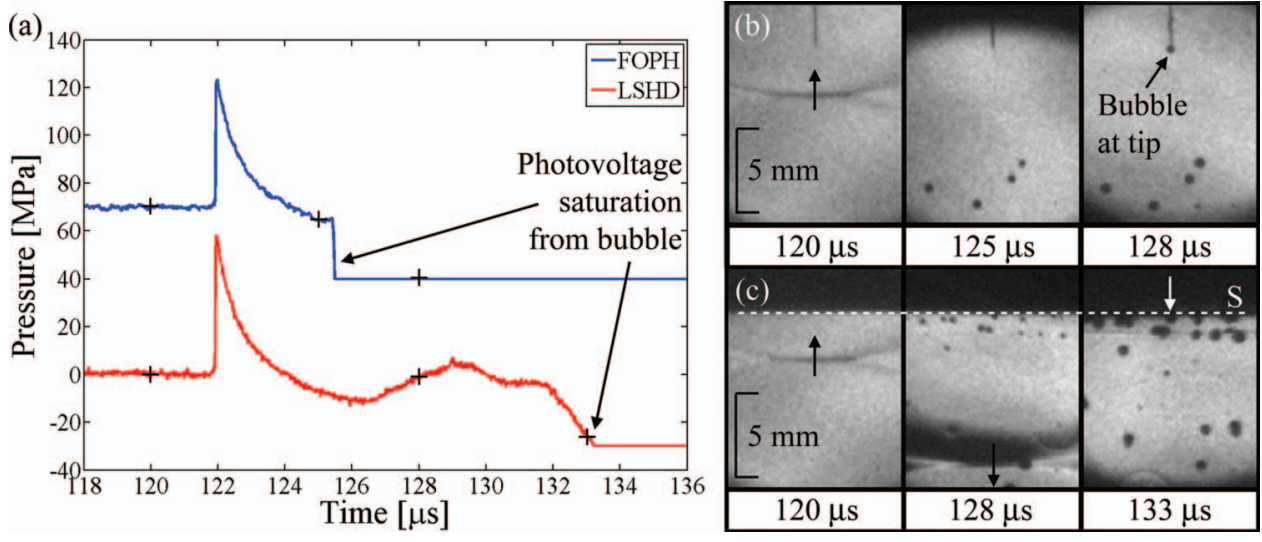

FIG. 8. (Color online) (a) Representative LSHD waveform (light) and FOPH waveform (dark; $+70 \mathrm{MPa}$ ) corrupted by bubbles at their respective measurement spots. Imacon 200 monitor output of respective camera exposures is indicated on waveforms (+). (b) FOPH and (c) LSHD bubble activity is shown besides corresponding waveforms. Arrows over lithotripter shock waves indicate propagation direction. White arrow on the final frame of (c) indicates approximate laser spot in imaging plane (determined from images of FOPH fiber tip alignment to focus). "S" marks the surface of the LSHD glass block. 
TABLE IV. Summary of benefits and drawbacks using the LSHD versus the FOPH.

LSHD

- Glass block translation away from damage area (no loss of alignment)

- No deconvolution necessary during SW duration; simple

-Separate baseline voltage channel for online calibration control

-Large, inflexible, and limited to in vitro studies

- LSW reflection induces greater cavitation activity

-Water level regulation needed to protect optics
FOPH

-Flexible geometry for a variety of measurement scenarios

- Near omnidirectional phase response.

- "Free field" lessens cavitation impact on measurements

Drawbacks

-Fiber break results in loss of alignment

-Deconvolution required for accurate calibration

-Laser overheating after extended use

\section{Hydrophone glass damage}

Cavitation contamination is unavoidable in either hydrophone, but the triviality of repeating measurements with a stable EM source makes this a minor issue unless bubble activity leads to permanent damage of the hydrophones' silica glass. When cavitation causes the FOPH fiber to break, the signal changes are unambiguous: dc baseline voltage in water will drop noticeably and subsequent data measurements may appear distorted or delayed depending on the nature of the break. When this occurs, the user must cut, clean, and reposition a new fiber.

The LSHD addresses glass damage-related issues with a unique screw translation feature that allows the user to relocate internal or glass surface flaws away from the laser spot focus while maintaining the point of measurement. When damage forms on the light spot or along the laser transmission lines, dc baseline approaches zero and measurements appear distorted from artifacts attributable to bubbles or internal laser light reflections. Damage formed nearby to the light spot may attract bubble activity capable of partial laser light reflection without visibly compromising the dc baseline. LSHD glass block translation can help to resolve these measurement distortions as well.

\section{Performance comparison and design considerations}

Certain differences in hydrophone measurements have been substantiated as cavitation effects, such as tensile wave shortening and acoustic radiation amassment. Further differences may result from unique photovoltage-to-pressure calibration processes. Inaccuracies in FOPH calibration during this study may have resulted from dc baseline voltage ambiguity during measurement and a light scattering calibration factor $(\alpha)$ that varies with optical fiber length. Error from changes in fiber losses has been estimated to be less than $4 \%$ over the course of FOPH measurement. In general, LSHD calibration errors can be caused by polynomial fitting approximations $(<1 \%)$ and nonexistent calibration adjustments for fiber optic losses or water temperature fluctuation, the latter of which contribute minimally $(<1 \%)$ to measurements in this study.

Table IV summarizes the main strengths and drawbacks inherent to the LSHD and FOPH. No mention is made of advantageous traits that both hydrophones share, such as those listed in Table I; both hydrophones have comparable spatial resolutions and large bandwidths that produced statistically similar shock front rise times ( $\sim 10 \mathrm{~ns})$ at the highest acoustic source settings. Additionally, both hydrophones are capable of rendering non-stop sequential high energy waveforms at a PRF up to $1 \mathrm{~Hz}$ without inordinate interference from bubbles.

The FOPH has one clear advantage over the LSHD in its application: flexibility. The very small and nearomnidirectional hydrophone has proven useful in a variety of arrangements that are inconceivable for the LSHD, including measurements in vivo and orthogonal to the acoustic propagation direction. For the LSHD, maintaining the glass block surface orthogonal to the shock wave propagation axis is critical for proper hydrophone calibration. As a result, problems arise in using the LSHD with steeply angled acoustic sources ( $>45^{\circ}$ relative to the horizon) because water can disrupt the fiber optic light guide paths. Pressure field mapping along the acoustic propagation $(z)$ axis requires water level adjustments from the user, and with non-zero acoustic source angles, measurements in the focal plane may too necessitate water level adjustment. Further refinement in the LSHD design should address waterproofing.

Because of the significant losses in its optical transmission-reception line, the FOPH needs a laser source with considerably more power than the LSHD laser (see Table I) to boost the signal-to-noise ratio. Overheating is a concern, as it reduces laser power, which lowers the dc baseline and increases measurement uncertainty. Additional FOPH redesign should consider improvements to its laser cooling system as well as implementation of separate ac and dc lines for simultaneous monitoring during waveform measurement.

\section{CONCLUSION}

As a supplement to the fiber optic probe hydrophone technology, the light spot hydrophone compensates for several FOPH limitations, such as loss of alignment from glass damage and complex calibration, although it poses a different set of drawbacks, such as inflexibility and the need for water level management. In lithotripter field measurements, both hydrophones adequately rendered standard parameters without unreasonable disruption from cavitation interference and damage, and both are suitable as focus and field hydrophones according to the international standard IEC 61846. For in vitro acoustic characterization, especially in open 
environments and highly variable shock sources, the LSHD can be a preferable alternative to the FOPH. In more confined and exacting setups, the FOPH remains a paragon among acoustic measuring devices.

\section{ACKNOWLEDGMENTS}

The authors would like to acknowledge the technical support provided by Dr. Yuri Pishchalnikov pertaining to hydrophone measurement distortion from low frequency filtration, and FOPH-related support provided by Professor Wolfgang Eisenmenger and Dr. Rainer Pecha. This work was supported by a research grant from Siemens. The use of the Imacon 200 high-speed camera was supported by National Institutes of Health (NIH) through Grant No. 510-RR016802.

${ }^{1}$ G. R. Harris, IEEE Trans. Ultrason. Eng. 39, 256 (1992).

${ }^{2}$ A. J. Coleman and J. E. Saunders, Ultrasound Med. Biol. 15, 213 (1989).

${ }^{3}$ J. Staudenraus and W. Eisenmenger, Ultrasonics 31, 267 (1993).

${ }^{4}$ Z. Q. Wang, P. Lauxmann, C. Wurster, M. Kohler, B. Gompf, and W. Eisenmenger, J. Appl. Phys. 85, 2514 (1999).

${ }^{5}$ G. R. Harris, IEEE Trans. Ultrason. Eng. 35, 87 (1988).

${ }^{6}$ B. Granz, IEEE Trans. Dielectr. Electr. Insul. 24, 499 (1989).

${ }^{7}$ R. L. Bedi and A. R. Selfridge, in IEEE Proceedings of Ultrasonics Symposium, Orlando, FL, 1991, Vol. 2, p. 1141.

${ }^{8}$ M. Platte, Ultrasonics 23, 113 (1985).

${ }^{9}$ G. Eberle and W. Eisenmenger, IEEE Trans. Dielectr. Electr. Insul. 27, 768 (1992).

${ }^{10} \mathrm{~J}$. Staudenraus and W. Eisenmenger, in Fortschritte der Akustik-DAGA '88, 1988, p. 467.

${ }^{11}$ P. Morris, A. Hurrell, A. Shaw, G. Gandhi, M. A. El-Sherif, and P. A. Lewis, J. Acoust. Soc. Am. 125, 3611 (2009).

${ }^{12}$ A. S. Daryoush, R. G. Minasamudram, P. Arora, G. Gandhi, M. A. ElSherif, and P. A. Lewis, Appl. Opt. 48, G77 (2009).

${ }^{13}$ W. Weise, V. Wilken, and C. Koch, IEEE Trans. Ultrason. Ferroelectr. Freq. Control 49, 937 (2002).

${ }^{14}$ F. Ueberle and A. Jamshidi-Rad, J. Phys.: Conf. Ser. 279, 6 (2011).

${ }^{15}$ X. Chavanne, S. Balibar, and F. Caupin, Phys. Rev. Lett. 86, 5506 (2001).

${ }^{16}$ P. A. Lewin, C. Mu, S. Umchid, A. Daryoush, and M. El-Sherif, Ultrasonics 43, 815 (2005).

${ }^{17}$ A. Arvengas, K. Davitt, and F. Caupin, Rev. Sci. Instrum. 82, 034904 (2011).

${ }^{18}$ Y. A. Pishchalnikov, O. A. Sapozhnikov, M. R. Bailey, I. V. Pishchalnikova, J. C. Williams, and J. A. McAteer, ARLO 6, 280 (2005)
${ }^{19}$ Y. A. Pishchalnikov, J. A. McAteer, R. J. Vonderhaar, I. V. Pishchalnikova, J. C. Williams, and A. P. Evan, J. Urol. 176, 2294 (2006).

${ }^{20}$ A. P. Evan, J. A. McAteer, B. A. Connors, Y. A. Pishchalnikov, R. K. Handa, P. Blomgren, L. R. Willis, J. C. Williams, J. E. Lingeman, and S. Gao, BJU Int. 101, 382 (2008).

${ }^{21}$ S. Zhu, T. Dreyer, M. Liebler, R. Riedlinger, G. M. Preminger, and P. Zhong, Ultrasound Med. Biol. 30, 675 (2004).

${ }^{22}$ G. N. Sankin, W. N. Simmons, S. L. Zhu, and P. Zhong, Phys. Rev. Lett. 95, 034501 (2005).

${ }^{23}$ E. Klaseboer, S. W. Fong, C. K. Turangan, B. C. Khoo, A. J. Szeri, M. L. Calvisi, G. N. Sankin, and P. Zhong, J. Fluid Mech. 593, 33 (2007).

${ }^{24}$ M. Arora, C. D. Ohl, and D. Lohse, J. Acoust. Soc. Am. 121, 3432 (2007).

${ }^{25}$ Y. A. Pishchalnikov, M. M. Kaehr, and J. A. McAteer, ASME International Mechanical Engineering Congress and Exposition (American Society of Mechanical Engineers, Seattle, WA, 2007), p. 1.

${ }^{26}$ A. Zijlstra and C. D. Ohl, J. Acoust. Soc. Am. 123, 29 (2008).

${ }^{27}$ P. V. Chitnis and R. O. Cleveland, J. Acoust. Soc. Am. 119, 1929 (2006).

${ }^{28}$ B. Granz, R. Nanke, J. Fehre, T. Pfister, and R. Engelbrecht, Med. Sol. 86, 86 (2004).

${ }^{29} \mathrm{G}$. Paltauf, H. SchmidtKloiber, and H. Guss, in Laser-Tissue Interaction and Tissue Optics (SPIE Press, 1996), Vol. 2923, p. 127.

${ }^{30}$ G. Paltauf and H. SchmidtKloiber, J. Appl. Phys. 82, 1525 (1997).

${ }^{31}$ V. A. Leitao, W. N. Simmons, Y. Zhou, J. Qin, G. N. Sankin, F. H. Cocks, J. Fehre, B. Granz, R. Nanke, G. M. Preminger, and P. Zhong, AIP. Conf. Proc. 900, 377 (2007).

${ }^{32}$ O. A. Sapozhnikov, V. A. Khokhlova, M. R. Bailey, J. C. Williams, J. A. McAteer, R. O. Cleveland, and C. A. Crum, J. Acoust. Soc. Am. 112, 1183 (2002)

${ }^{33}$ P. Huber, K. Jochle, and J. Debus, Phys. Med. Biol. 43, 3113 (1998).

${ }^{34}$ M. Liebler, T. Dreyer, and R. E. Riedlinger, Ultrasonics 44(Suppl. 1), e319 (2006).

${ }^{35}$ G. N. Sankin and V. S. Teslenko, Dokl. Phys. 48, 665 (2003).

${ }^{36}$ D. V. Voronin, G. N. Sankin, V. S. Teslenko, R. Mettin, and W. Lauterborn, J. Appl. Mech. Tech. Phys. 44, 17 (2003).

${ }^{37}$ C. D. Ohl, Phys. Fluids 14, 3512 (2002).

${ }^{38}$ D. L. Sokolov, M. R. Bailey, and L. A. Crum, J. Acoust. Soc. Am. 110, 1685 (2001)

${ }^{39}$ J. I. Iloreta, N. M. Fung, and A. J. Szeri, J. Fluid Mech. 616, 43 (2008).

${ }^{40}$ M. L. Calvisi, J. I. Iloreta, and A. J. Szeri, J. Fluid Mech. 616, 63 (2008).

${ }^{41}$ J. E. Parsons, C. A. Cain, and J. B. Fowlkes, J. Acoust. Soc. Am. 119, 1432 (2006).

${ }^{42}$ G. N. Sankin, Y. F. Zhou, and P. Zhong, J. Acoust. Soc. Am. 123, 4071 (2008).

${ }^{43}$ E. N. Harvey, A. H. Whiteley, W. D. McElroy, D. C. Pease, and D. K. Barnes, J. Cell. Comp. Physiol. 24, 23 (1944).

${ }^{44}$ B. M. Borkent, M. Arora, and C. D. Ohl, J. Acoust. Soc. Am. 121, 1406 (2007).

${ }^{45}$ B. M. Borkent, S. Gekle, A. Prosperetti, and D. Lohse, Phys. Fluids 21 (2009). 